\title{
Status of recent developments in collision avoidance using motion detectors based on insect vision
}

\author{
D. Abbott, A. Moini, A. Yakovleff * X.T. Nguyen, R. Beare, W. Kim † A. Bouzerdoum, \\ R.E. Bogner, K. Eshraghian $\ddagger$ \\ Centre for High Performance Integrated Systems and Technologies (CHiPTec) \\ The University of Adelaide, SA 5005, Australia \\ email: dabbott@eleceng.adelaide.edu.au
}

\begin{abstract}
Insects tend to detect motion rather than images and this together with inherent parallelism in their visual architecture, leads to an efficient and compact means of collision avoidance. A VLSI implementation of a smart microsensor that mimics the early visual processing stage in insects has been developed. The system employs the 'smart sensor' paradigm in that the detectors and processing circuitry are integrated on one chip. The IC is ideal for motion detection, particularly collision avoidance tasks, as it essentially detects the speed, bearing and time-to-impact of a moving object. The Horridge model for insect vision has been directly mapped into VLSI and therefore the IC truly exploits the beauty of nature in that the insect eye is so compact with parallel processing, enabling compact motion detection without the computational overhead of intensive imaging, full image extraction and interpretation. This world-first has exciting applications in areas such as anti-collision for automobiles and autonomous robots.
\end{abstract} VLSI

Keywords: Photodetectors, smart sensors, insect vision, collision avoidance, biologically inspired engineering,

\section{INTRODUCTION}

For collision avoidance tasks, where full imaging is unecessary, simple motion detection of object boundaries offers an efficient solution. A world-first single-chip device, based on insect vision principles, has been developed ${ }^{1-6}$ that outputs the time-to-impact, bearing and velocity of a detected object. The processing power of a commercial microcontroller is then sufficient for making decisions based only on such simple variables.

The insect vision model we have adopted is that of Horridge, ${ }^{7,8}$ which is named the 'template model.' This model is motivated by the desire to produce signals which can be readily interpreted by digital systems, and hence

\footnotetext{
*With the Defence Science and Technology Organisation (DSTO), Information Technology Division, Salisbury, SA 5108, Australia

tWith Seoul National University, Kwanak-gu, Shinlim-dong, 151-742, South Korea

$\ddagger$ Also with Edith Cowan University, Joondalup, WA 6027, Australia
} 


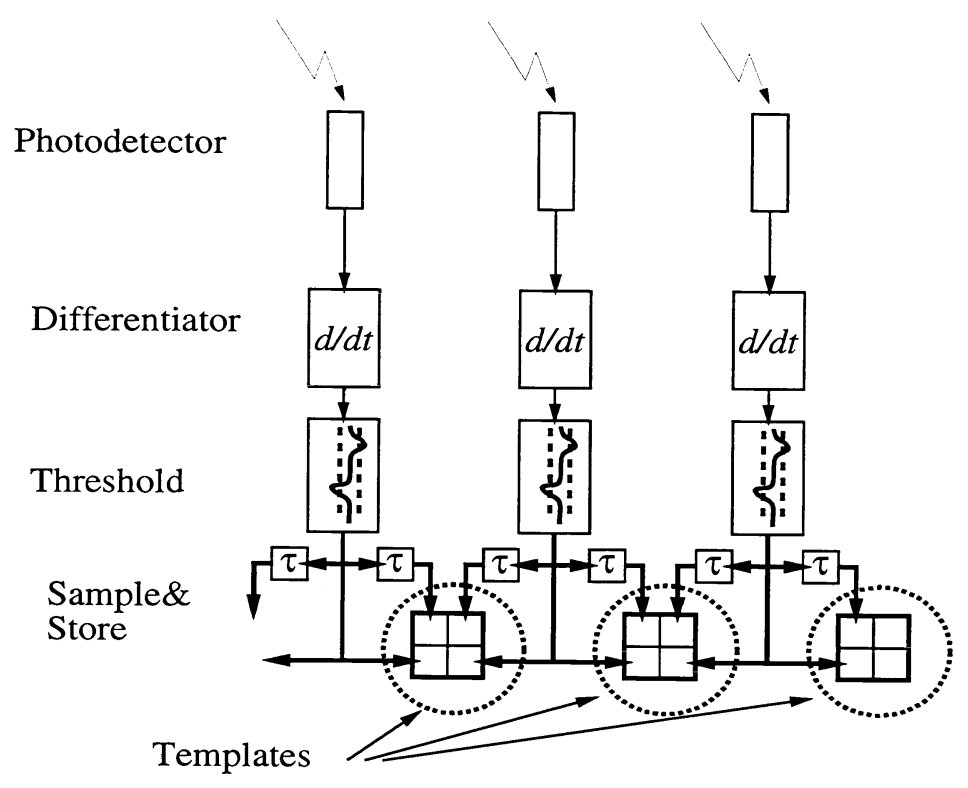

Figure 1: Template Model Functional Blocks

readily lended itself for mapping onto a VLSI chip. The edge of an object presenting a difference in contrast with the background, and moving in front of an array of receptors, elicits distinctive patterns of contrast changes which are consistent with the direction of motion. The receptor outputs are sampled and compared with their previous values, yielding signals which locally indicate an increase, decrease, or no change in contrast. The combination of two adjacent receptor responses at consecutive sampling times form a 'template,' and hence, since there are three possible receptor responses, there are 81 possible templates. The VLSI implementation and detailed description of this scheme is described elsewhere.

In this paper, we firstly review the template model and compare this with the biological insect eye architecture. We then proceed to discuss some of the implementation problems and developments that have occured to solve them.

\section{OVERVIEW OF INSECT VISION VERSUS TEMPLATE MODEL}

The advantage of a smart-sensor that can mimic insect vision is that the image processing is simplified and can be integrated on the detector chip, creating a compact device ideal for mobile applications. In addition, insects operate with no iris action or focusing adjustment required - this is also a feature of the developed sensor - leading to a truly solid-state vision system. Such simplicity is an important factor for high-volume robotic/automotive applications.

The chip accepts a real-time optical image and indicates the motion of edges in the visual field. From the outputs of the chip, we can infer the bearing, time-to-impact, and speed of objects in the visual environment.

Fig. 1 shows the concept of the Template Model. Light is detected by photoreceptors, a temporal differentiation of the signal takes place, the signal is thresholded and then two samples (separated in time by $\tau$ ) are combined 


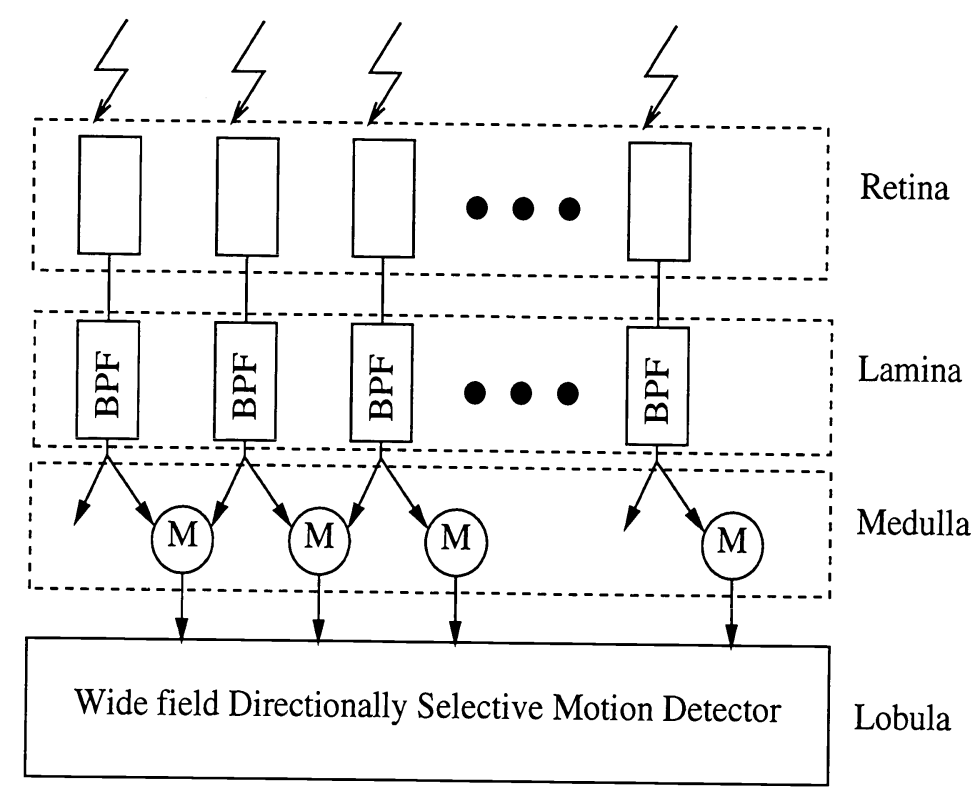

Figure 2: Simplified Model of the Insect Visual System

with those of an adjacent channel to form a 2 by 2 spacio-temporal template.

On the other hand, Fig. 2 shows the biological architecture of insect vision. The photodetection layer is called the retina and the individual segments of the compound eye are called omatidia. The next layer is called the lamina and this performs a band pass filter (BPF) function to select higher frequencies or alternatively it can more simply be a high pass filter (HPF). This function can be thought of as a coarse temporal differentiation - when a signal is differentiated it is the high frequency edges that are passed through. Hence, in the Horridge model this is represented as a differentiator. The purpose of this stage is to detect temporal changes in contrast (motion). The next layer, called the medulla, detects changes between adjacent omatidia - hence local motion is detected. The Horridge model does the same, however, makes a departure from biology, at this point, by considering digitally thresholded data. The final lobula layer represents the 'intelligence' and this analyses the local contrast changes and decides more globally whether motion has taken place over a wider field. This section is not dealt with in the template model. The template model only goes as far as the medulla.

In our physical VLSI implementation, the receptors are simply p-well/n-epi junction photodetectors. Light is focussed by means of a GRIN lens and the photocurrent is converted to a voltage by a subthreshold circuit technique that guarantees a logarithmic photoresponse. The logarithmic response to light intensity allows us to dispense with the need for an iris, which is not present in insect vision and is not specifically addressed by the template model. This function can be regarded as an auto gain control (AGC) mechanism.

Temporal differentiation is physically achieved by an operational transconductance amplifier (OTA) based differentiator. The time constants required to mimic insect vision are in the order of $10 \mathrm{~ms}$. Thus to avoid the problem of a large capacitance area for the differentiator, it turns out that a feedback resistance in the order of 1 Giga-Ohm is required! This creates quite a design challenge and a number of active resistor configurations have been tried, including one based on the channel length modulation effect.

The next stage is template formation where the signal is simply thresholded, sampled and stored, and hence a template consists of the current and and stored outputs of adjacent channels. The chosen sampling rate is in the order of a $100 \mathrm{~Hz}$, which is adequate for analog VLSI, and also comparable with the fastest time constant of 


\begin{tabular}{|l|l|l|l|}
\hline Function & Biological Model & Template Model & Physical/VLSI Implementation \\
\hline Photodetection & Retina (omatidia) & Receptor & $\begin{array}{l}\text { - pn junction photodiode } \\
\text { GRIN lens } \\
\text { - Subthreshold I-V converter } \\
\text { (logarithmic AGC) }\end{array}$ \\
$\begin{array}{l}\text { Temporal Contrast } \\
\text { Change Detection }\end{array}$ & Lamina (BPF) & $\begin{array}{l}\text { Temporal } \\
\text { differentiator }\end{array}$ & OTA differentiator \\
$\begin{array}{l}\text { Local Motion } \\
\text { Detection }\end{array}$ & Medula & $\begin{array}{l}\text { Template } \\
\text { formation }\end{array}$ & $\begin{array}{l}\text { Thresholding, sample } \\
\text { and store circuitry }\end{array}$ \\
$\begin{array}{l}\text { Wide Field Motion } \\
\text { Detection }\end{array}$ & Lobula & N/A & $\begin{array}{l}\text { Template tracking- software } \\
\text { control via microcontroller }\end{array}$ \\
\hline
\end{tabular}

Table 1: Comparison of IC implementation with biological and template models.

insect motion detection neurons. ${ }^{9}$

Now that templates are formed, they can be simply matched (hence the name 'template') against a look up table to interpret the direction of motion locally to the receptors that generated that template.

The next stage is to perform the function of the lobula and interpret the local motion information to select an overall motion over a wider field. Here the Horridge model stops and does not deal with this issue. The way we physically extract overall motion information from clusters of templates is basically via software control of an external microcontroller. The insect vision chip loads template information into an external memory and an off-the-shelf microcontroller performs various 'template tracking' algorithms to extract the bearing, speed and time-to-impact of an approaching object. ${ }^{10-13}$

The comparison between the biological insect eye architecture, the template model and our physical IC implementation, in terms of required function is summarized in Table. 1.

\section{STATUS AND FUTURE DIRECTION}

Table 2 indicates the chronological development and future vision for our insect vision chip dubbed the 'bugeye.' Bugeye I of 1992 vintage was the first design for proof-of-concept and it contained both analog and digital circuitry. The signal was differentiated for detecting changes in contrast. It successfully operated with bearing, velocity and time-to-impact being successfully extracted from the template output. However, the drawback was that it only worked under DC light sources. The Bugeye II redesign produced a chip with improved dynamic range and contained a multiplicative noise cancellation circuit (MNC). ${ }^{16}$ The digital sections of the chip were discarded in favor of an external microcontroller. The MNC circuit allowed the chip to successfully operate under AC lighting conditions. The principle of MNC is to simply divide the signal in each channel by the spatial average over a number of channels. The circuit is designed so the averages over 3,5 or 7 channels can be externally selected. As the detected signal luminance $L$ is simply a function of the reflectance of an object $\rho$ times the illuminance of the incident light $E$, division by the spatial average cancels the $\mathrm{E}$ terms (containing the unwanted $\mathrm{AC}$ noise component), resulting in a simple ratio of reflectances or contrast ratio. This has three benefits: (1) reduction in the effect of the $50 \mathrm{~Hz}$ or $60 \mathrm{~Hz}$ hum from AC light sources, (2) a data compression due a simple contrast ratio figure producing numbers close to unity and (3) and edge enhancement due to a reduction in spatial average near the edges. 


\begin{tabular}{|l|l|l|l|}
\hline Version & Size & Technology & Year \\
\hline Bugeye I & 64 by 12 & $2 \mu$ m CMOS & 1992 \\
Bugeye II & 64 by 2 & $1.2 \mu$ m CMOS & 1994 \\
Bugeye III & 64 by 32 & $0.8 \mu$ m CMOS & 1996 \\
Bugeye IV & 64 by 64 & BiCMOS & 1997 \\
Bugeye V & 64 by 64 & GaAs or CGaAs & 1998 \\
\hline
\end{tabular}

Table 2: Evolution of 'Bugeye' Insect Vision Chip

The latest Bugeye III design contains a truly 2 dimensional array of detectors. AGC occurs at every node by virtue of logarithmic compression due to subthreshold detector circuits, however contrast change detection is carried out by only one row of differentiators. This is achieved by clocking signal out of the device as in conventional $2 \mathrm{D}$ arrays.

Bugeye IV will be an array of a larger size and perhaps exploit the qualities BiCMOS, being an analog device. Bugeye $\mathrm{V}$ will be a proof-of-concept in GaAs. This can be in a conventional E/D MESFET process or in the newly emerging complimentary CGaAs process.

In order to minimise design risks, it was deemed preferable at the start to employ a 'stable' and well-behaved technology, and so far each IC has been fabricated in CMOS. However recent developments indicate that GaAs may eventually become a viable alternative to $\mathrm{CMOS}^{6}$ The main advantage is that the photoresponse of GaAs is superior to CMOS in terms of performance and spectral properties.

In addition to developing better circuits, the optical interface should not be neglected. The GRIN lens used until now imposes certain restrictions on the design. A relatively new technology, called 'binary optics,' needs investigation and is beginning to be successfully employed. This technology consists of integrating tiny microlenses on the surface of the IC.

\section{CONCLUSION}

We have reviewed our physical IC implementation of a motion detector, based on the template model, and have carefully outlined each function with reference to the biological architecture of insect vision.

The first chip contained a linear array of 60 photodetectors. A second generation of this device contained 60 by 2 detectors and multiplicative noise cancellation (MNC) to reduce the effects of hum from AC lighting, producing edge enhancement and carrying out a form data compression. Current work is extending the present concept to a 2-D array and future work will exploit the many advantages of a gallium arsenide implementation and binary optics.

\section{ACKNOWLEDGEMENTS}

This work has been generously funded by the Australian Research Council (ARC), the Department of Employment Education and Training (DEET) and Britax-Rainsfords P/L. A special thanks is due to Emeritus Professor G. A. Horridge for his inspiration. 


\section{REFERENCES}

[1] A. Moini, A. Bouzerdoum, A. Yakovleff, D. Abbott, O. Kim, K. Eshraghian, and R. E. Bogner, "An analog implementation of early visual processing in insects," International Symposium on VLSI Technology, Systems, and Applications, Taipei, pp. 283-287, May 12-14, 1993.

[2] A. Yakovleff, A. Moini, A. Bouzerdoum, X. T. Nguyen, R. E. Bogner \& K. Eshraghian, "A micro-sensor based on insect vision," Conference on Computer Architectures for Machine Perception (CAMP'g3), New Orleans, USA, pp. 137-146, 15-17 Dec. 1993.

[3] A. Bouzerdoum, R. E. Bogner, K. Eshraghian, A. Moini, A. Yakovleff \& X. T. Nguyen, "A VLSI frontend visual processor based on insect vision," Sensory Stratagems: From Living Eyes to Seeing Machines, Canberra, 7-11 Feb. 1993.

[4] D. Abbott, A. Moini, A. Yakovleff, X.T. Nguyen, A. Blanksby, G. Kim, A. Bouzerdoum, R.E. Bogner \& K. Eshraghian, "A new VLSI smart sensor for collision avoidance inspired by insect vision," Proc. SPIE, Boston, Vol. 2344, pp. 105-115, Nov. 2-4, 1994.

[5] A. Yakovleff, D. Abbott, X.T. Nguyen \& K. Eshraghian, "Obstacle avoidance and motion-induced navigation," Proc. Comp. Arch. for Machine Perception (CAMP'95), Como, Italy, pp. 384-393, 18-20 Sep. 1995.

[6] D. Abbott, A. Yakovleff, A. Moini, X.T. Nguyen, A. Blanksby, R. Beare, A. Beaumont-Smith, G. Kim, A. Bouzerdoum, R.E. Bogner and K. Eshraghian, "Biologically inspired obstacle avoidance - a technology independent paradigm," Proc. SPIE, Philadelphia, Vol. 2591, pp. 2-12, 22-23 October 1995.

[7] G. A. Horridge, "The compromise between seeing spatial layout and making visual discriminations," Current Science, Vol. 60, No. 12, pp. 686-693, June 1991.

[8] G. A. Horridge, "Ratios of template responses as the basis of semivision," Phil. Trans. Roy. Soc. London B, Vol. 331, pp. 189-197, 1991.

[9] K. Hausen, "Motion sensitive interneurons in the optomotor system of the Fly-II," Biological Cybernetics, Vol. 46, pp. 67-79, 1982.

[10] A. Yakovleff, X. T. Nguyen, A. Bouzerdoum, A. Moini, R. E. Bogner, K. Eshraghian, "Dual-purpose interpretation of sensory information," Proc. IEEE Int. Conf. Robotics and Automation, San Diego, Vol. 2, pp. 1635-1640, 8-13 May, 1994.

[11] X. T. Nguyen, A. Yakovleff, A. Moini, K. Eshraghian, A. Bouzerdoum, R. E. Bogner, "VLSI architecture of a low computational load processor for a visual system based on insect vision," European design \& test conf., pp. 85-89, 1994.

[12] X. T. Nguyen, A. Bouzerdoum, R.E. Bogner, K. Eshraghian, D. Abbott, A. Moini, "The stair-step tracking algorithm for velocity estimation," ANZIIS-93, Perth, pp. 412-416, 1-3 Dec., 1994.

[13] X. T. Nguyen, A. Bouzerdoum, A. Yakovleff, A. Moini, R. E. Bogner, K. Eshraghian, "VLSI robotic microsensor: range-finder from self motion," IEEE Proc. mechatronics and machine vision in practice, Toowoomba, pp. 78-83, 13-15 Sep., 1994.

[14] A. Bouzerdoum, A. Moini, A. Yakovleff, X. T. Nguyen, R. E. Bogner, K. Eshraghian, "A smart visual micro-sensor," Proc. IEEE Int. Conf. on Systems, Man and Cybernetics, 2-5 Oct. 1994.

[15] X. T. Nguyen, K. Eshraghian, A. Moini, A. Bouzerdoum, A. Yakovleff, D. Abbott, R. E. Bogner, "An implementation of a smart visual micro-sensor based upon insect vision," 12th Australian Microelectronics Conference, Queensland, pp. 129-134, 5-8 Oct., 1993.

[16] A. Moini, et al, "Multiplicative Noise Cancellation (MNC) in Analog VLSI Vision Sensors," IEEE Proc. Elec. Tech. Directions to the Year 2000, Adelaide, Australia, pp. 253-257, May 23-25, 1995. 